\title{
On the wrong side of history
}

\author{
As anti-vaccination movements gain momentum in some regions, World Immunization Week 2017 \\ reminds us that vaccines work and are safe, and that ideological positions contrary to this truth ignore \\ the weight of scientific evidence and deny a long history of lives being saved, endangering many more.
}

Ever since the demonstration by

Edward Jenner in the late eighteenth century that inoculating humans with material from cowpox-infected cows provided protection against smallpox, individuals and organized groups have opposed vaccination, based on safety, political or religious concerns. Jenner's initial methods, which involved the scoring of children's flesh to inoculate them with pus from a cowpox blister, drew opposition from both parents who disapproved of the need to injure their child, and the clergy who criticized the use of an animal source as "unchristian". But even decades later, when safer vaccines were made available and the UK Parliament instituted the Vaccination Act of 1853 - ordering the mandatory vaccination of infants and introducing penalties against parents failing to do so - wider protests erupted focusing on the "infringement of personal liberty and choice", including a demonstration in Leicester in 1885 that gathered up to 100,000 people $^{1}$. Such protests culminated in a new Vaccination Act in 1898 that removed penalties and introduced a conscience clause, allowing parents who did not believe in vaccine safety or efficacy to obtain a certificate of exemption. Notably, such non-medical exemptions still exist today and their frequency is steadily increasing in some US states, despite links to outbreaks of infectious diseases, such as measles ${ }^{2}$.

Recently, several 'controversies' have been exploited by anti-vaccine advocates, including alleged links between DTP (diphtheria, tetanus and pertussis) immunizations and neurological conditions; and the MMR (measles, mumps and rubella) vaccine and thimerosal (a mercury-containing preservative included in some vaccine formulations) with autism ${ }^{3}$. Importantly, many of the studies supporting such links have been discredited and/or retracted most famously Andrew Wakefield's study suggesting a link between the MMR vaccine and autism - and several subsequent studies have disproved any link between vaccination or thimerosal and neurological or behavioural disorders ${ }^{3}$. Nonetheless, such claims and their widespread media coverage have dented confidence in vaccination among sections of the public, leading to decreased vaccine uptake and coverage in multiple countries, culminating in outbreaks of preventable diseases such as pertussis and measles in countries like Ireland, Japan, the Netherlands, Nigeria, Sweden, the UK and the US ${ }^{2,4}$.

Vaccine opposition continues today, as demonstrated by the latest Open Challenge from the World Mercury Project (http:// go.nature.com/2mOuJDw), spearheaded by Robert F. Kennedy and Robert De Niro, which will pay US\$100,000 to anyone who can demonstrate that "thimerosal is safe in the amounts contained in vaccines currently being administered to American children and pregnant women". The announcement, which follows a recent meeting between Kennedy and President Trump - who has publicly supported the links between vaccines and autism ${ }^{5}$ - illustrates how baseless concerns about vaccine safety are still being whipped up among the public, while credible scientific information is often ignored, such as a US Institute of Medicine report that found no evidence between thimerosal (and the MMR vaccine) and autism ${ }^{6}$.

In light of these developments, this year's theme for World Immunization Week, Vaccines Work, is timely and appropriate, although could perhaps send a stronger message if changed to: Vaccines are Safe and Work. Paradoxically, vaccine success is probably another reason for the decrease in vaccine coverage observed in some industrialized countries. For example, the introduction of efficacious vaccines in the twentieth century in the US has virtually eliminated deaths caused by diphtheria, tetanus, poliomyelitis, smallpox, measles, mumps, rubella and Haemophilus influenzae type B meningitis. Furthermore, vaccination of US children born during 1994-2013 has been estimated to have prevented 322 million illnesses, 21 million hospitalizations and 732,000 deaths during their lifetimes ${ }^{7}$. Therefore, while previous generations witnessed first-hand the debilitating and often life-threatening effects of diseases such as polio, smallpox, measles, tetanus and rubella, newer generations in these countries perceive them as uncommon and rarely fatal. However, although some diseases like smallpox have been eradicated, and others like polio are on the verge of eradication, several vaccine-preventable diseases such as tetanus, measles and rubella are still amongst the leading causes of child mortality and morbidity worldwide. Measles, for example, is estimated to have been responsible for 134,200 deaths globally in 2015, whereas neonatal tetanus killed around 49,000 newborn children in 2013, and over 100,000 babies are born with congenital rubella syndrome every year. Such examples highlight that vaccines remain essential, and that vaccine $R \& D$ must remain a priority in global healthcare agendas.

There are also reasons for renewed optimism, as demonstrated by the recent introduction of a new vaccine against dengue virus in endemic regions; by promising results in malaria vaccines undergoing clinical trials; and by a phase III clinical trial in Guinea showing an Ebola vaccine to be safe and effective ${ }^{8}$. Furthermore, governments and funding agents have come together to address problems identified during the latest epidemics, leading to the establishment of the Coalition for Epidemic Preparedness Innovations, whose mission is to coordinate the development of new vaccines9.

Despite this progress, it is important to remind ourselves that vaccines against major pathogens such as HIV and Mycobacterium tuberculosis are still missing or afford incomplete protection, and that even for vaccine-preventable diseases, populationlevel protection still depends on attaining worldwide vaccine coverage rates that enable herd immunity and prevent outbreaks. Therefore, different aspects of vaccination campaigns (including information, awareness, vaccine storage and deployment) must not be neglected. What a waste it would be to have vaccines that are safe and work, but misinformed people that refuse to use them. $\square$

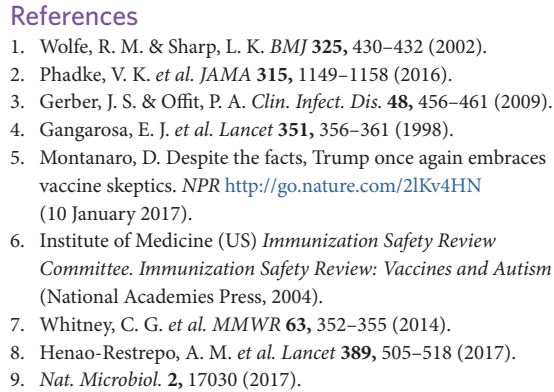

